on the Council of the Royal Entomological Society and a Vice President in 1962. His services were similarly used in education where he was chief examiner of a number of organizations, a committee member of the Education Subcommittee of the Zoological Society and on the Biological Education Committee of the Royal Society and Institute of Biology. He was made a Fellow of the Institute of Biology in 1967.

As a scientist he travelled widely, and in 1962 lectured at the Royal Ontario Museum. He visited Warsaw in 1964 through the hospitality of the Polish Academy of Sciences, and was invited to the University of Louvain in 1967.

These achievements, however, represented only some of the facets of the man, for more than anything else John Carthy realized the relationships of science to other studies. His interests in art, drama and literature sprang from the same source as the critical faculties, understanding, humour and patience which he gave so freely to his colleagues in science.

\section{Professor Louis Joel Mordell}

Professor L. J. Mordell, the acknowledged doyen of British number theory, died on March 12, 1972, at the age of 84.

Louis Mordell was born an American citizen in Philadelphia in 1888 . Attracted to mathematics by books in a second-hand store, he noted that many of the problems were taken from Cambridge Scholarship and Tripos papers, so, in 1906, he crossed the Atlantic alone to sit the scholarship examination of the St John's group and was awarded a major scholarship. He took Part I of the Mathematical Tripos in 1909, the last year of the published order of merit, and was third after P. J. Daniell and E. H. Neville. He remained in Cambridge until 1912, when he was awarded the second Smith's Prize. After periods at Birkbeck College, the Ministry of Munitions and Manchester College of Technology, he became Fielden Professor of Mathematics in the University of Manchester in 1923 and was elected to the Royal Society in 1924 . A capable and conscientious head of department, he built up a strong school. In 1945 he left Manchester to succeed G. H. Hardy in the Sadleirian Chair in Cambridge. After his retirement in 1953 he retained his base in Cambridge but travelled much and was for several years a visiting professor in the USA and Canada. He retained his passionate interest in mathematics and mathematicians unabated and continued to publish and to lecture on his work right to the end.

Mordell's most famous individual achievement is his "finite basis theorem". This deals with rational points on algebraic curves (that is, with points $(x, y)$ with rational coordinates which satisfy the equation $f(x, y)=0$ of the curve). Algebraic curves are classified geometrically by a non-negative integer, the genus. For curves of genus zero the properties of the rational points were completely elucidated at the turn of the century. For curves of genus greater than one, there is still no theory of any generality, though much work has been motivated by Mordell's conjecture that there are at most finitely many rational points. It is curves of genus one which have the greatest interest today since there is an extensive theory as well as plausible but recalcitrant conjectures: and it is here that Mordell's theorem is central. Poincaré had already shown that the rational points on a curve of genus one have a natural group structure. Mordell showed in 1922 that, as conjectured by Poincaré, this group always has a finite basis. Weil generalized this to abelian varieties.

Throughout his life Mordell was fascinated by Diophantine equations of all kinds (that is, the solution of equations in rationals, or, alternatively, in integers) and he displayed a quite remarkable virtuosity. His only substantial book, written when he was already an octogenarian, is a valuable account of this fascinating but little systematized area. A particular favourite was the determination of all the integral points $(x, y)$ on the curve $y^{2}=x^{3}+k$, where $k \neq 0$ is a given integer. Special cases had been considered already by Fermat and others but the equation is sometimes known as "Mordell's equation". The Smith's Prize essay of 1912 developed several lines of attack and determined all the solutions for many values of $k$. At the time Mordell believed it to be possible that there could be infinitely many solutions for certain $k$, but he subsequently realized that his work, together with a then little known result of Axel Thue showed that the number was always finite. The finiteness proof was, however, nonconstructive; that is, there was the logical possibility that one could never be sure whether a given set of solutions was complete or not, and so it remained a challenging problem to find complete sets of solutions. In 1947 Mordell chose $y^{2}=x^{3}+k$ as the subject for his Cambridge Inaugural Lecture, which was published by the University Press under the title $A$ Chapter in the Theory of Numbers. In a sense this chapter was closed by Alan Baker who, as a special case of his general theory, has given an effective procedure for finding all the solutions for given $k$, albeit one that requires multilength computer programs for its practical implementation.

Rather similar to Diophantine equa- tions is the theory of polynomial congruences, which occupied Mordell at several stages of his career. The problem here is to study or estimate the number of solutions of a congruence $f\left(x_{1}, \ldots, x_{n}\right) \equiv 0(\bmod p)$, where $f$ is a polynomial with integer coefficients and $p$ in the most interesting cases is a prime. Mordell brought his habitual ingenuity and dexterity to bear. His earlier results were superseded by deep estimates of Hasse and Weil but he returned to the subject later and showed that his techniques were still valuable in a more general context. Similar remarks apply to Mordell's contributions to the estimation of trigonometric sums.

Mordell was an expert in modular forms and functions. In 1917 he proved one of the famous conjectures of Ramanujan about his "tau-function", the coefficients of a certain modular form. Later Hecke was to show that the property in question follows from those of the "Hecke operator" and is a special case of a general phenomenon. Mordell used modular theory to investigate certain peculiar recurrence relations between the class numbers of imaginary quadratic forms which had been discovered by Kronecker, Liouville and others and showed that they were best understood as resulting from equating coefficients in identities between modular forms. He also used modular theory to investigate the number of representation of integers as sums of squares, particularly the more difficult case where the numbers of squares is odd.

Another of Mordell's major interests was the Geometry of Numbers. Not merely did he contribute himself but he sparked off the renaissance of the subject in the 1940's and 1950's associated in addition with the names of Mahler, Davenport and Rogers. Perhaps his most notable result was the determination of the lattice constant of the region $|f(x, y)|<1$, where $f$ is a binary cubic form.

No brief account can do justice to the variety and interest of Mordell's work. His achievements and influence rank him with the masters of the theory of numbers.

\section{Announcements}

\section{International Meetings}

May 1-3, Breeding of Endangered Species, St Helier (The Secretary, Jersey Wildlife Preservation Trust (ESI), Les Augrès Manor, Trinity, Jersey, Channel Islands).

May 8, Movement for Survival, London (Michael Schwab, Department of Nutrition, Queen Elizabeth College, London W8 7AH). 